\title{
Improving efficiency and lowering operating costs of evaporative cooling
}

\author{
Jan Taler ${ }^{*}$, Bartosz Jagieła ${ }^{2}$, and Magdalena Jaremkiewicz ${ }^{1}$ \\ ${ }^{1}$ Cracow University of Technology, Department of Power Engineering, Al. Jana Pawla II 37, 31-864 \\ Cracow \\ ${ }^{2}$ Cracow University of Technology, Department of Thermal Processes, Air Protection, and Waste \\ Utilisation S-5, ul. Warszawska 24, 31-155 Cracow
}

\begin{abstract}
Cooling towers, or so-called evaporation towers, use the natural effect of water evaporation to dissipate heat in industrial and comfort installations. Water, until it changes its state of aggregation, from liquid to gas, consumes energy $(2.257 \mathrm{~kJ} / \mathrm{kg})$. By consuming this energy, it lowers the air temperature to the wet-bulb temperature, thanks to which the medium can be cooled below the ambient temperature. Evaporative solutions are characterized by continuous water evaporation (approx. 1.5\% of the total water flow) and low electricity consumption (high EER). Evaporative (adiabatic) cooling also has a positive effect on the reduction of electricity consumption of cooled machines. Lowering the relative humidity $(\mathrm{RH})$ by approx. $2 \%$ lowers the wet-bulb temperature by approx. $0.5^{\circ} \mathrm{C}$, which increases the efficiency of the tower, operating in an open circuit, expressed in $\mathrm{kW}$, by approx. $5 \%$, while reducing water consumption and treatment costs. The use of the M-Cycle (Maisotsenko cycle) to lower the temperature of the wet thermometer to the dew point temperature will reduce operating costs and increase the efficiency of cooled machines.
\end{abstract}

Keywords: M-Cycle; evaporation tower; cooling tower; adiabatic; IEC; Indirect Evaporative Cooling; DEC; Direct Evaporative Cooling; dew point cooling

\section{Introduction}

Polish industry sectors are responsible for approx. $40 \%$ of energy consumption, i.e. 65 TWh (data from the Central Statistical Office for 2019 [1]). An estimated half of this power is used for thermal processes (including pumps, compressors, fans, etc...). Lowering this value can be achieved by modernizing worn-out installations or building new energy-saving ones, using the best available techniques [2].

All industrial sectors, such as air conditioning, refrigeration and power production, emits heat. Some of this energy can be recovered and reused, e.g. for heating of water. The remainder of the useless heat must be dissipated due to its contents low amount of energy. Industrial cooling are systems in which excess heat is removed from the medium (water,

\footnotetext{
* Corresponding author: bartosz.jagiela@doktorant.pk.edu.pl
} 
glycol, refrigerant, etc.) while reducing its temperature to the ambient temperature. Most often, heat is utilized with water and/or air to the outside environment.

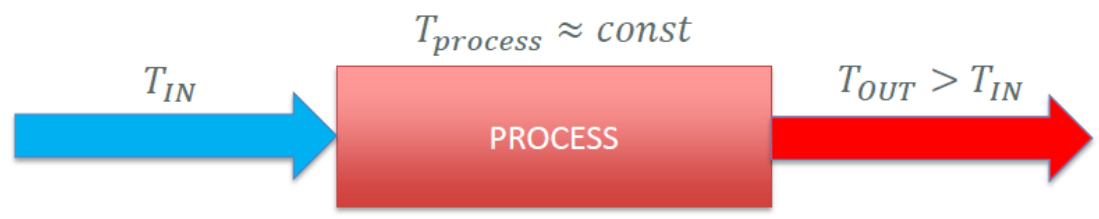

Fig. 1. Energy flow in industry processes [3].

A cooling system should be designed and applied in a way that will ensure appropriate technological conditions, start-up, operation and stoppage of the machines most effectively and durably. Local factors limit design possibilities. Such factors include climate, water availability, space available for structures, and the location and related restrictions, such as noise emissions or environmental pollution.

One of the most important factors is the local climate as it directly affects the final temperature of the medium or air used as a coolant. Refrigeration equipment is designed so that the maximum cooling capacity is achieved under the most unfavourable environmental conditions. These temperatures are the dry thermometer and the wet thermometer, which depends on the air humidity.

Depending on which temperature, wet or dry, we design a cooling system, we distinguish between dry and wet cooling, also known as evaporative cooling. Additionally, one can distinguish between adiabatic and hybrid cooling, a combination of dry and wet cooling.

The psychometric graph shows the reference points for each cooling method, including dry cooling in red, evaporative cooling in blue and adiabatic cooling in orange. The arrow indicates the initial parameters that are constant for each and are as follows:

- The temperature of dry-bulb: $+35^{\circ} \mathrm{C}$,

- Relative humidity: $35 \%$.

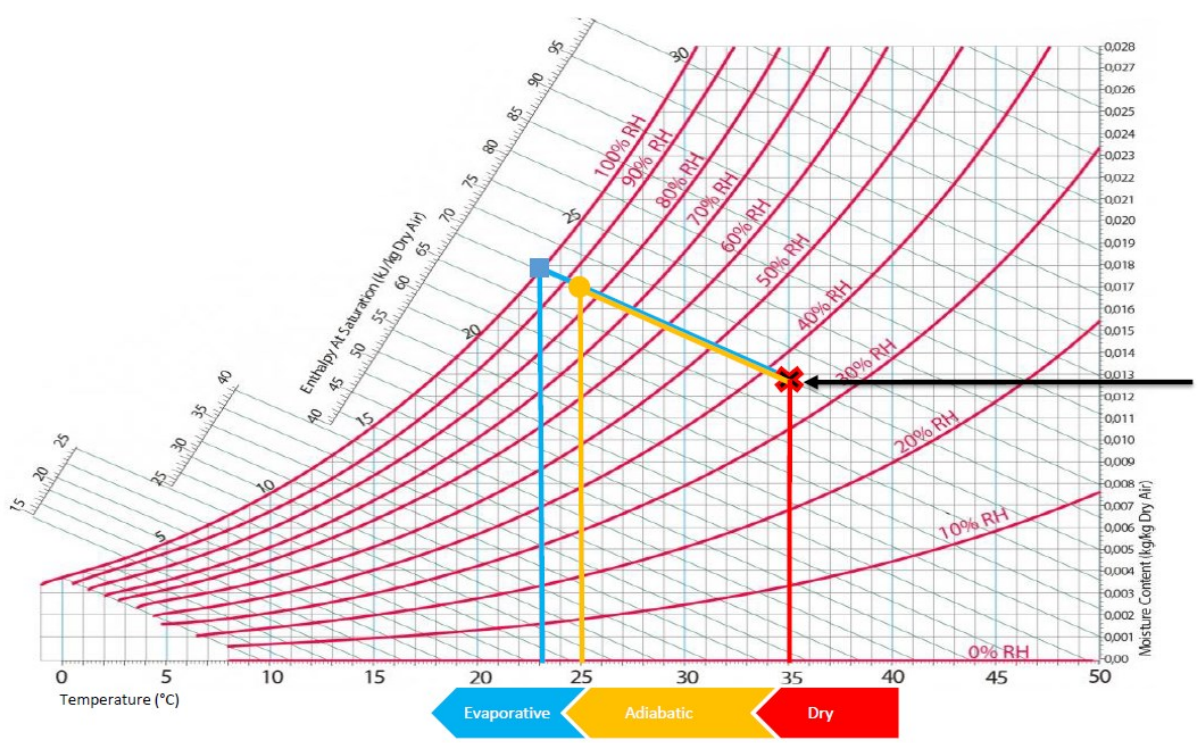

Fig. 2. Boundaries of each cooling type shown on the psychrometric diagram [3].

Although conventional air-conditioning (compressor cycle) systems are more likely accepted and installed, even of high power consumption, they cover a significant part of the 
market. Research and developments should not only focus on more effective compressors (also other equipment) in terms of power usage. Another point is the impact on our planet. Most refrigerants which are used in air-conditioning systems are harmful to the environment or people. Most sustainability refrigerant is water. That is why evaporative air-conditioning is a really promising technology and should be more likely to use. This paper will investigate the potential of using a combination of different evaporative cooling conceptions [4-6].

\section{Energy consumption in a refrigeration cycle}

Direct and indirect energy consumption is an important aspect of designing appropriate equipment and complete refrigeration systems. The coefficient of performance (COP) of such a system is expressed as the difference between the electricity consumed and the heat produced (energy of cooling). The refrigeration systems implement a thermodynamic cycle, also the so-called Linde cycle, the efficiency of which is expressed by the pressure difference between the evaporator and the condenser.

To increase efficiency, including the reduction of energy consumption, the pressure difference between the condenser and the evaporator should be reduced. In cooling or airconditioning systems, the evaporation temperature of the medium is constant, depending on the medium or reservoir being cooled. To lower the condensing pressure, a device that is capable to lower the condensing temperature below ambient temperature should be used. An example of such a solution is evaporative towers.

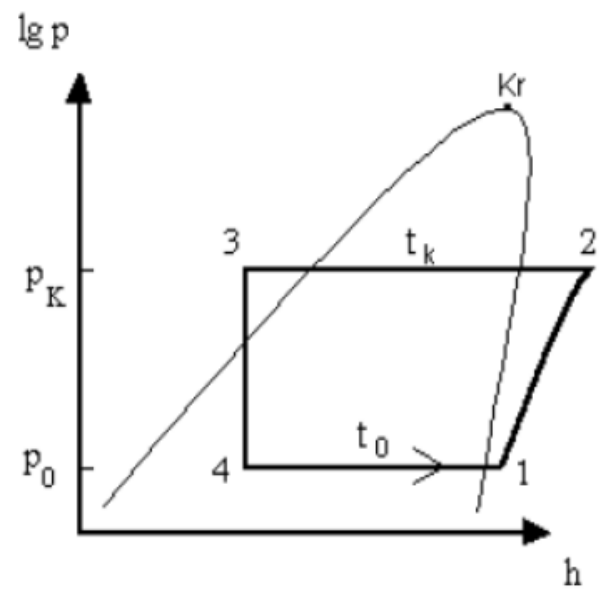

Fig. 3. Caption of the Figure 1. Below the figure.

\section{Evaporative cooling}

Evaporative cooling stands out by high thermal efficiency, thanks to the use of the natural effect of water evaporation, with minimal energy consumption and recirculation of approx. $95 \%$ of water. Evaporative technologies are able the process temperature to be reduced below ambient temperature (dry thermometer). Compared to dry air cooling, evaporative cooling is more effective because 1 kilogram of water can absorb $2.200 \mathrm{~kJ}$ of heat (evaporation heat), while 1 kilogram of air only approx. $1 \mathrm{~kJ}$ per $1 \mathrm{~K}$. 


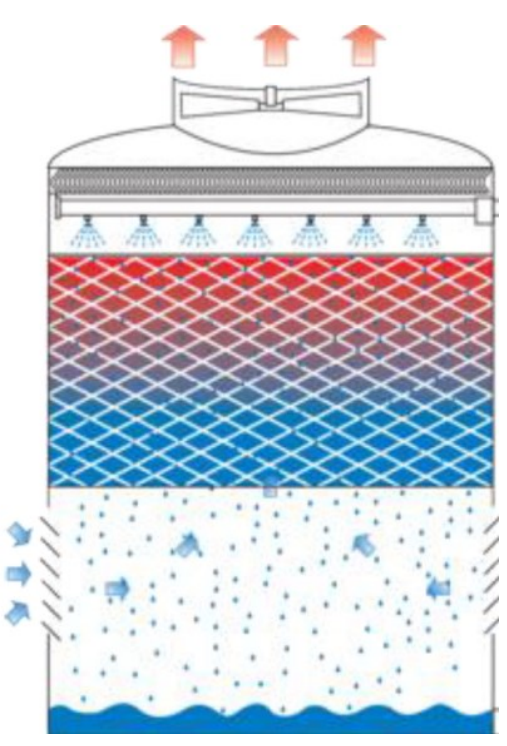

(a)

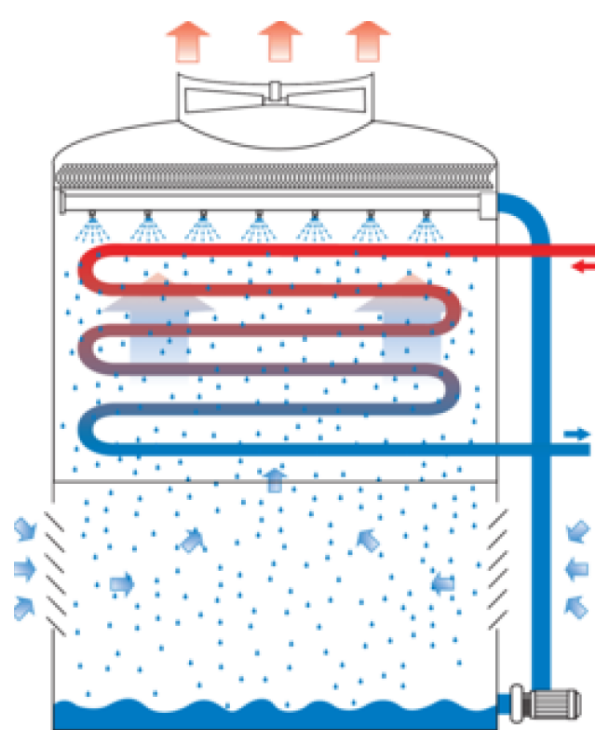

(b)

Fig. 4. Example of (a) open and (b) close cooling tower [7].

\subsection{Water usage}

Cooling towers are characterized by the continuous consumption of water while the device is in operation. Contrary to popular knowledge, water consumption does not depend on the type of device but external parameters and the quality of water in the circuit. Figure 5 shows the water flow balance in a closed evaporation tower.

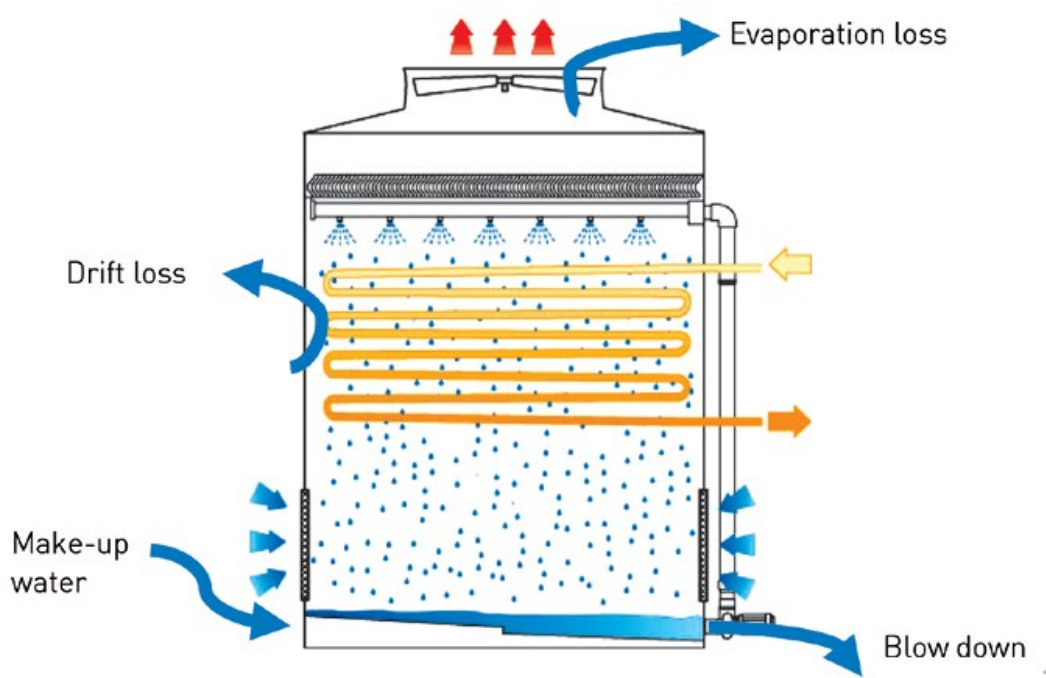

Fig. 5. Water balance of close cooling tower [8].

Main water flows are:

- Losses:

- evaporation loss, 
- drift loss,

- blowdown,

- Make-up water.

The water flow balance, in the cooling tower loop, should be preserved and can be expressed as:

$$
\text { Make up = Evaporation loss }+ \text { Drift loss }+ \text { Blow down }
$$

Water that is sprinkled on a special filling (air-water channels, open tower) or the exchanger (closed tower) evaporates with the airflow. The value of this loss is a few percent of the water flow and can be estimated as follows:

$$
V_{w}=\frac{Q_{c}}{594}
$$

594 - is the mean value of the latent heat of evaporation divided by the specific heat $\mathrm{kcal} / \mathrm{kg}$ water. The real value is related to humidity and the wet-bulb thermometer. This relationship is depicted in Figure nr 6.

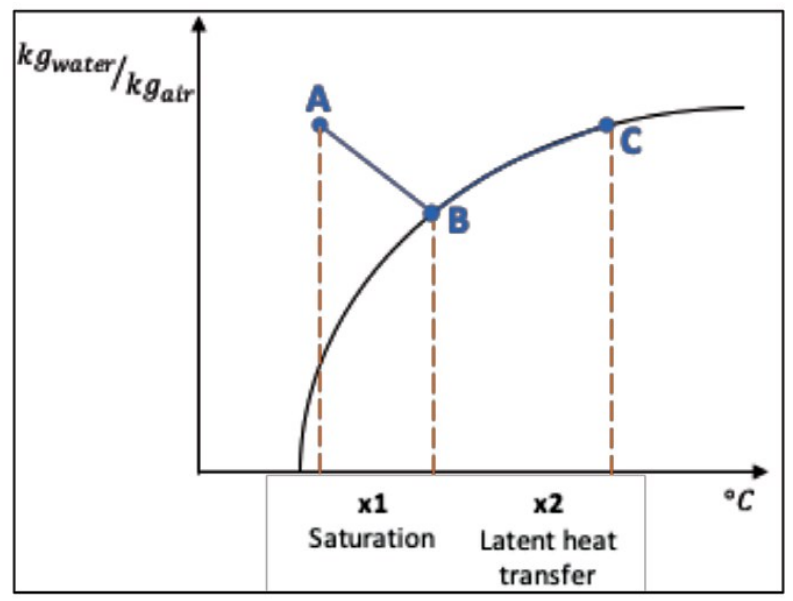

Fig. 6. Evaporation losses [8].

Another loss is blowing down, due to desalination of water (desludging), which is aimed at refreshing and restoring the appropriate parameters of the circulating water in the cooling tower loop. The exhaust air from the cooler is $100 \%$ humid and contains pure H2O. The remaining minerals, including salt (conductivity), hardness, chlorides and $\mathrm{pH}$, remain in the water basin. With evaporation, the parameters change, increasing their concentration. Desalination is aimed at the discharge of such an amount of water that the water parameters do not exceed the maximum values for the appropriate materials used in the installation. The value of the desalination water discharge depends primarily on the quality of the water and the water treatment system used. Knowing the water inlet parameters, an appropriate number of concentration cycles can be designed. Having this value determined, the desalination value can be calculated from the formula [8]:

$$
\text { Blow down }=\frac{\text { Evaporation loss }}{C-1}
$$

The aim is to maintain the concentration cycles in the range from 2 up to 4 because investment and operating costs above this value are not economical compares to savings.

The smallest loss in evaporative coolers is the drift loss. In the balance of water consumption, practically negligible because drift eliminators are used above the nozzles, which reduce drift to approx. $0.001 \%$ of the evaporation value. 


\section{Comparison of dry and wet cooling systems}

As indicated above, the use of evaporative coolers will have a positive effect on the efficiency of the cooling system with a simultaneously increased effort to keep the equipment in operation, in particular, proper water preparation.

Two refrigeration systems were simulated using CoolPack software [9]. One with an aircooled condenser (dry cooling) and another one with an evaporative condenser (wet cooling). To analyse the operation of these refrigeration systems, the following parameters were adopted:

- Refrigerant: R134a

- Cooling capacity: $1000 \mathrm{~kW}$

- Evaporation temperature: $+2^{\circ} \mathrm{C}$

- Dry bulb temperature: $+35^{\circ} \mathrm{C}$

- Relative humidity: $36 \%$

- Wet-bulb temperature: $+23^{\circ} \mathrm{C}$

- Isentropic efficiency of compression: 0.7

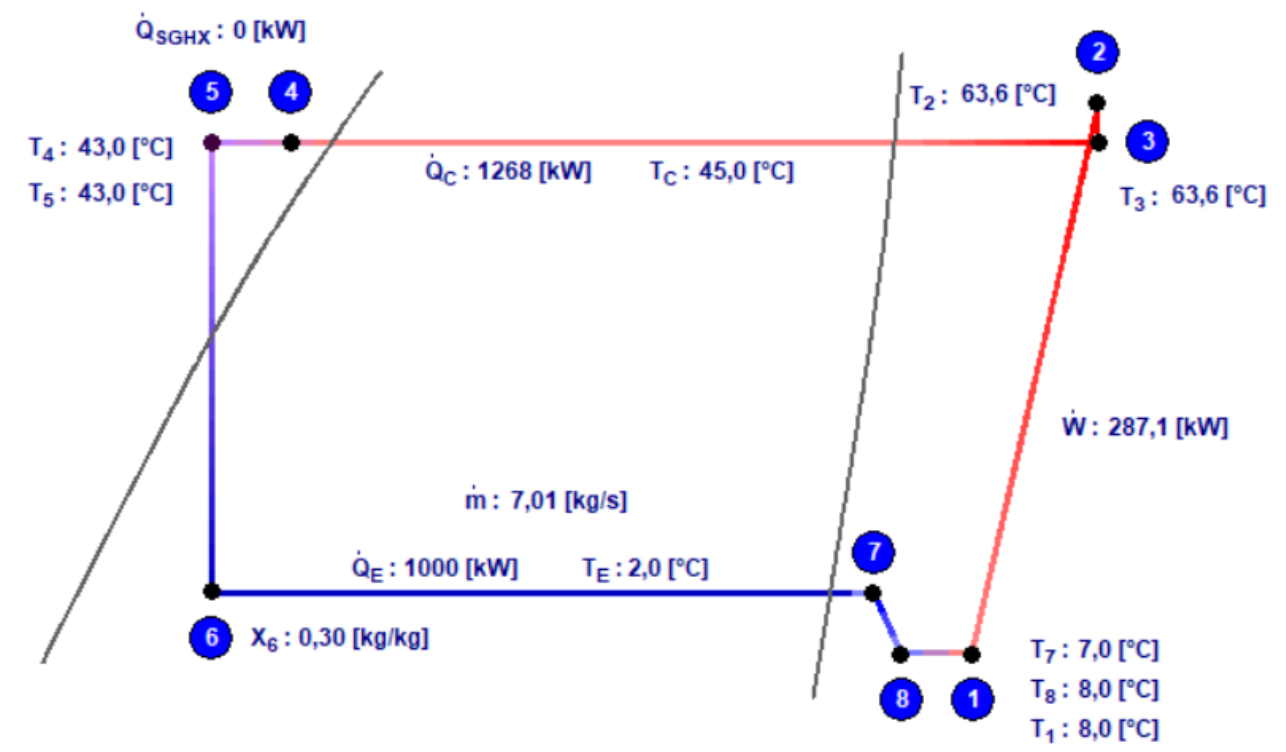

Fig. 7. Refrigeration cycle with air-cooled condenser [9]. 


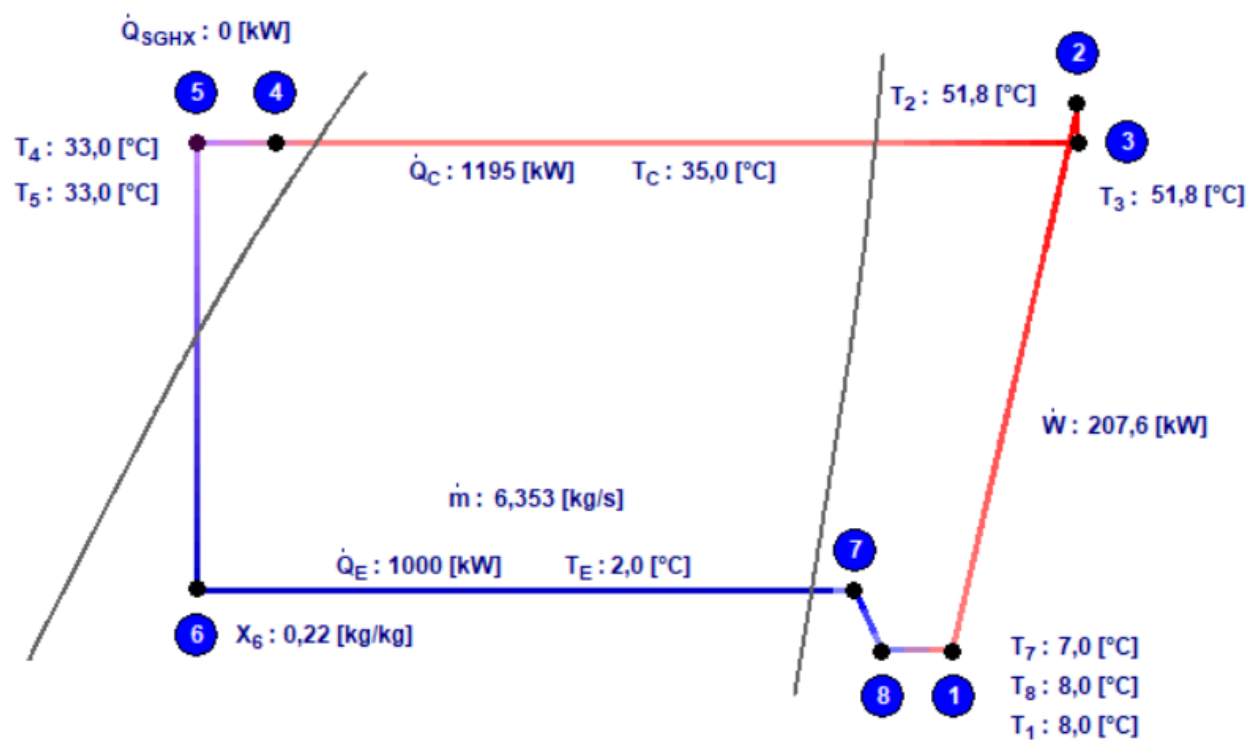

Fig. 8. Refrigeration cycle with evaporative condenser [9].

Figures 7 and 8 show the basic working conditions of each cycle. Most of the parameters of the wet cooling system are more favourable than those of the dry one. In particular:

- Condenser capacity (heating capacity) is approximately $6 \%$ lower - condenser could be smaller,

- Compressor input is around $28 \%$ lower - energy consumption and size of the compressor will be smaller,

- Mas flow of refrigerant is approximately $10 \%$ less - pipes and equipment could be smaller, - COP - Coefficient of Performance - higher by $39 \%$.

\section{Direct Evaporation Cooling (DEC)}

As previously indicated, the efficiency of the evaporation system depends on the temperature of the dry air and its humidity. The lower the humidity value, the lower the wet-bulb temperature, the higher the efficiency of the device. A good case of using water evaporation technology is the so-called evaporative air conditioners. On a special panel (cellulose), water flows crosswise into the air, which lowers its temperature to the temperature of the wet thermometer.

This type of device is commonly used for direct air cooling, mainly for air conditioning large spaces, such as production halls. Evaporators provide fresh and cool air with low energy and water consumption. The energy consumption of such a solution is about $85 \%$ lower than that of air conditioning with compressors. 


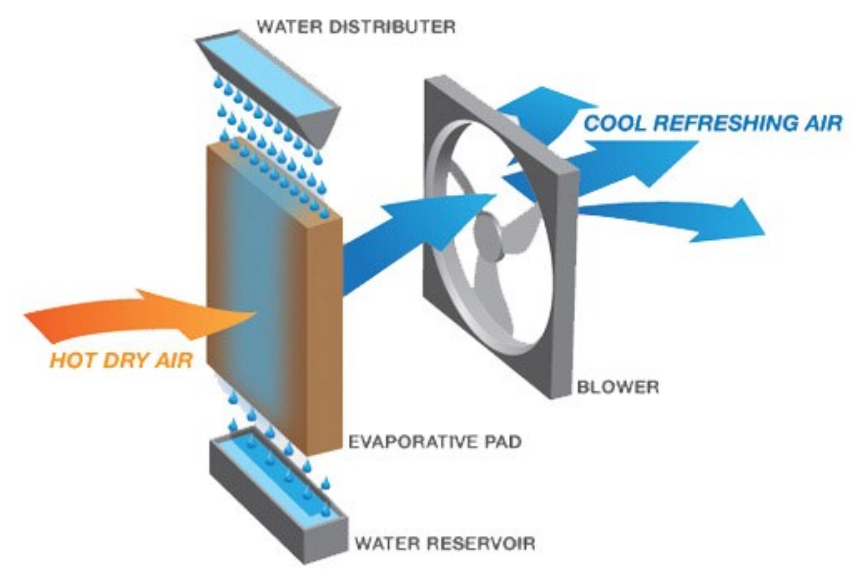

Fig. 9. Example of DEC [10].

\section{Indirect Evaporative Cooling - IEC, Maisotsenko Cycle - M- Cycle}

The concept of the M-Cycle was created in the seventies in the Soviet Union by Professor Valeriy Maisotsenko. In 1976 the first patents were issued (SU979796 and SU620745). Since then, the M-Cycle has been used in many applications, most commonly in the USA, which are protected by patents, the most important are:US4350570 (1982); US4842052 (1989); US4971245 (1990); US4976113 (1990); US4977753 (1990); US5453223 (1995); US5812423 (1998); US5838587 (1998); US6497107 (2002); US6581402 (2003); US6705096 (2004); US20040103637 (2004); US6776001 (2004); US6779351 (2004); US6854278 (2005); US6948558 (2005); US7007453 (2006); US7197887 (2007); US7228699 (2007); and US8613839 (2013). Multi-industry solutions were patented, including water distillation, power engineering (gas turbines, engines, energy and exergy towers) and pollution control (i.e. NOx reduction in gas turbines) [11].

The most popular solution and commercialized by Coolerado (USA) is the use of the MCycle for direct cooling of the air. The Maisotsenko cycle is a thermodynamic process that absorbs energy from the air, using the psychrometric (air humidity) renewable energy available from the latent heat of water evaporating into the air. It combines the thermodynamic processes of heat exchange and evaporative cooling to enhance obtaining the temperature of the dew point of the air. In other words, the M-Cycle achieves a dew point temperature (approx. $18.2^{\circ} \mathrm{C}$ for parameters from figure 2 ) which is lower than the wet-bulb temperature. Figure 10 shows the difference between DEC and IEC [11]. In Figure 10, the following designations have been adopted: Twb - wet-bulb, Tdp - dew point, 1 - inlet air conditions, 2 - outlet air conditions, 3 - outlet air conditions of working airflow.

The principle of operation of M-Cycle is based on paths of dry and wet channels. The scheme is shown in figure 11. Cross-flow heat and mass exchanger (HMX) is built of layers that are connected each to another forming a "box".

Air is divided into two streams, working and product. Each enters a different path. Product, so flow which will be cool, is flowing through dry channels. Working flow, which will evaporate water, is cooled under constant humidity. No mass is exchanged in dry channels. The working stream is entering wet channels precooled, temperature lower than ambient, which allows evaporating rest of the water. That activates latent heat which is absorbed by dry channels. The product becomes cooler and cooler. Streams at end of the channel are $50 \%$ of the flows which enters previously. 


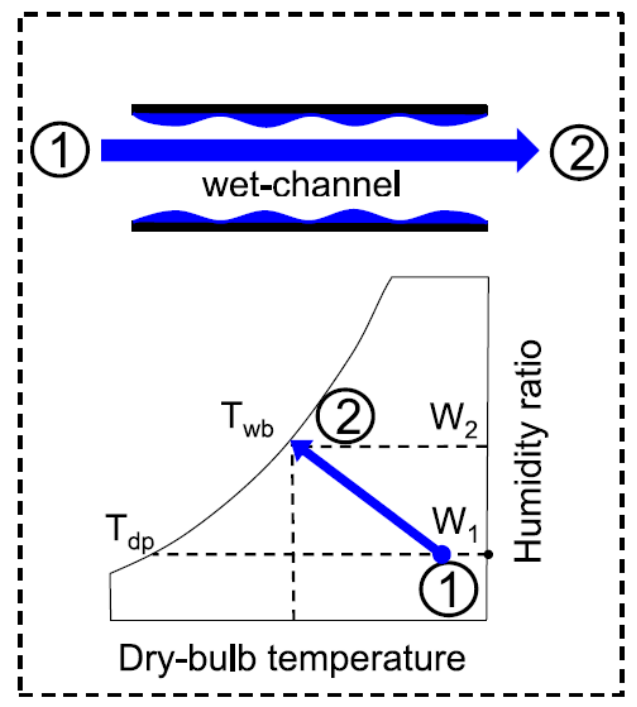

(a)

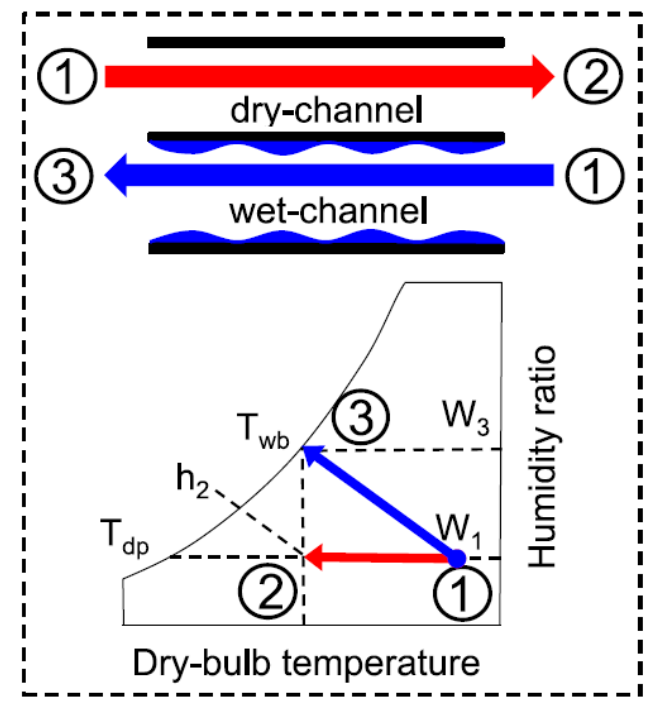

(b)

Fig. 10. Principle of operation of standard evaporative cooling techniques [11]: (a) DEC, (b) IEC.

Another benefit that M-Cycle applies is a fact that the product is keeping the same humidity. It is achieved by layer construction of HMX and also smart heat and mass transfer ratio.

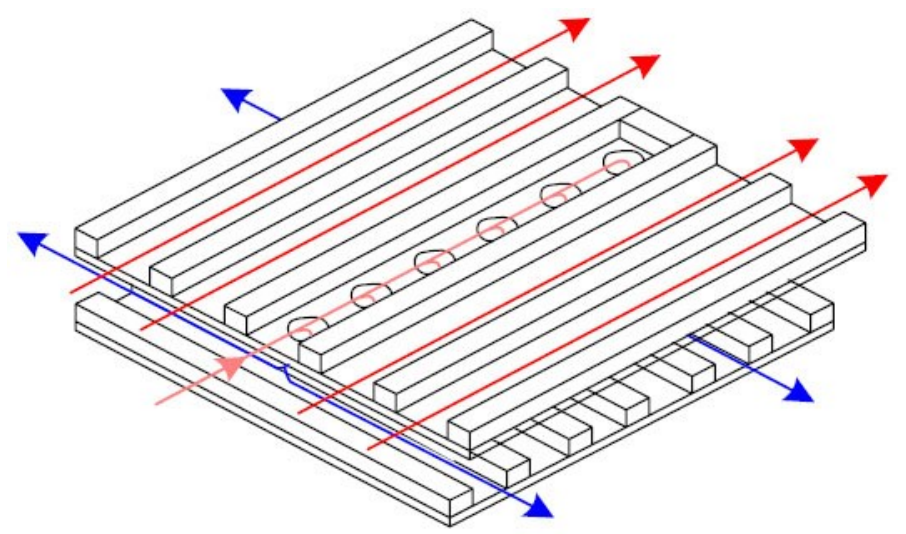

Fig. 11. Heat and mass exchanging in HMX [12].

In the paper [12] there was investigated how low temperature can be reached by M-Cycle in Mediterranean climate. The region is hotter and more humid than the polish one. It was proved that even in high ambient temperature, like $+42.1^{\circ} \mathrm{C}$, it was possible to reach leaving air temperature $\left(+19.2^{\circ} \mathrm{C}\right)$ close to dew point $\left(+18.5^{\circ} \mathrm{C}\right)$.

Dew point evaporative cooling have great potential to use it in HVAC technologies. Not only indirect cooling of air. One and still not developed is a connection of M-Cycle with the evaporative cooling tower. Precooled air leaving M-Cycle can enhance the effectiveness of cooling towers in terms of lowering reference temperature from wet-bulb up to dew point. If we make an evaluation similar as in figures 7 and 8 , but taking into consideration lower condensation temperature we will obtain working condition, as shown in Figure 12. 


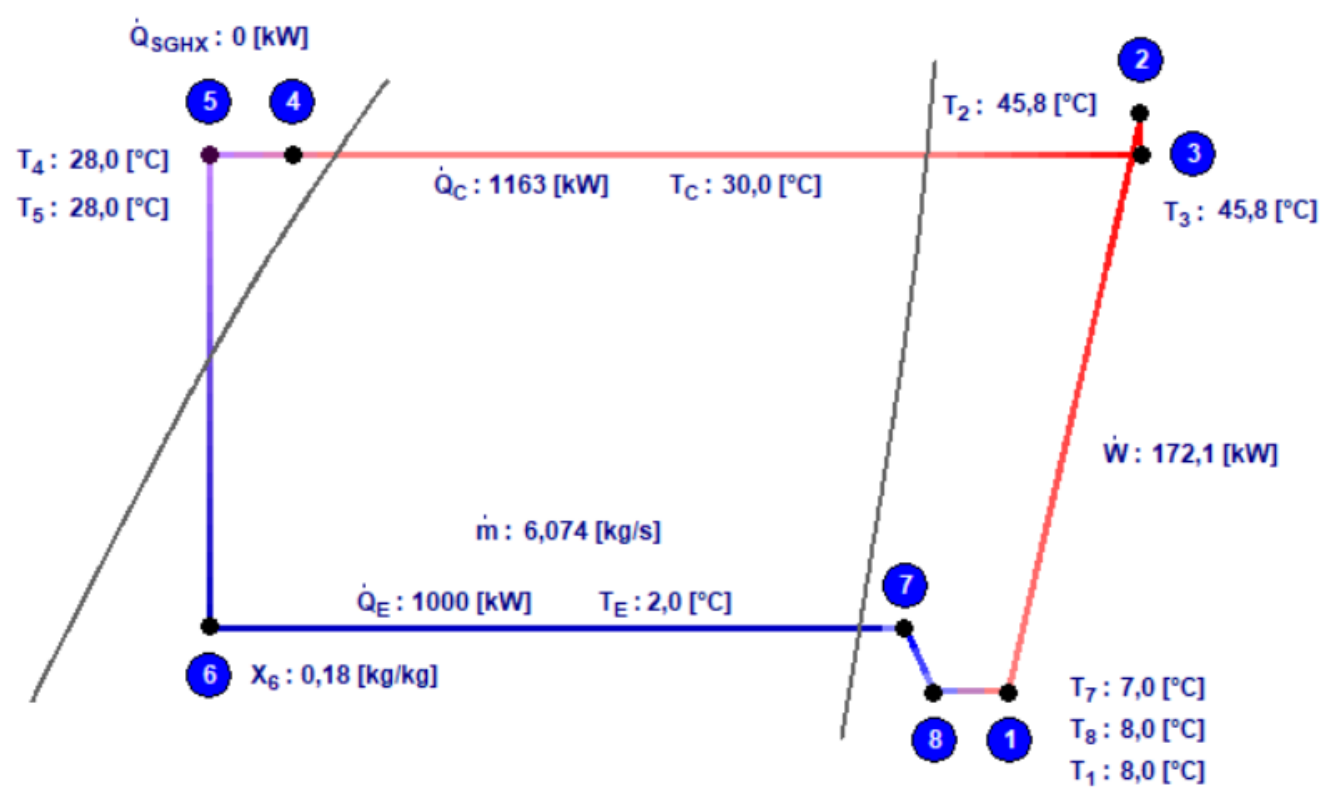

Fig. 12. Refrigeration cycle with dew point cooling [9].

Figure 12 shows how the refrigeration cycle will change its parameters when dew point cooling will be applied. It may be concluded that (compares to dry cooling):

- Condenser capacity (heating capacity) is more than $9 \%$ lower,

- Compressor input is around $41 \%$ lower,

- Mas flow of refrigerant is approximately $14 \%$ less,

- COP - Coefficient of Performance - higher by $67 \%$.

The presented three evaluations were shown on the lgp-h diagram in Figure 13. It is easily visible that the pressure difference between condenser and evaporator can be from $\Delta p$ $=3.6$ bar up to $\Delta \mathrm{p}=2.5$ bar. That gives the possibility to use smaller equipment which will consume less energy.

The present paper provides an overview of evaporative cooling and M-Cycle and its applicability in comfort and industry sectors. Maisotsenko cycle combines thermodynamic process, heat transfer and evaporative cooling, which is capable to enhance the effectiveness of air cooling. This conception is characterized by low energy and water usage. The HVAC application of M-Cycle is well known and commercialized but its applicability is still undrawn. 


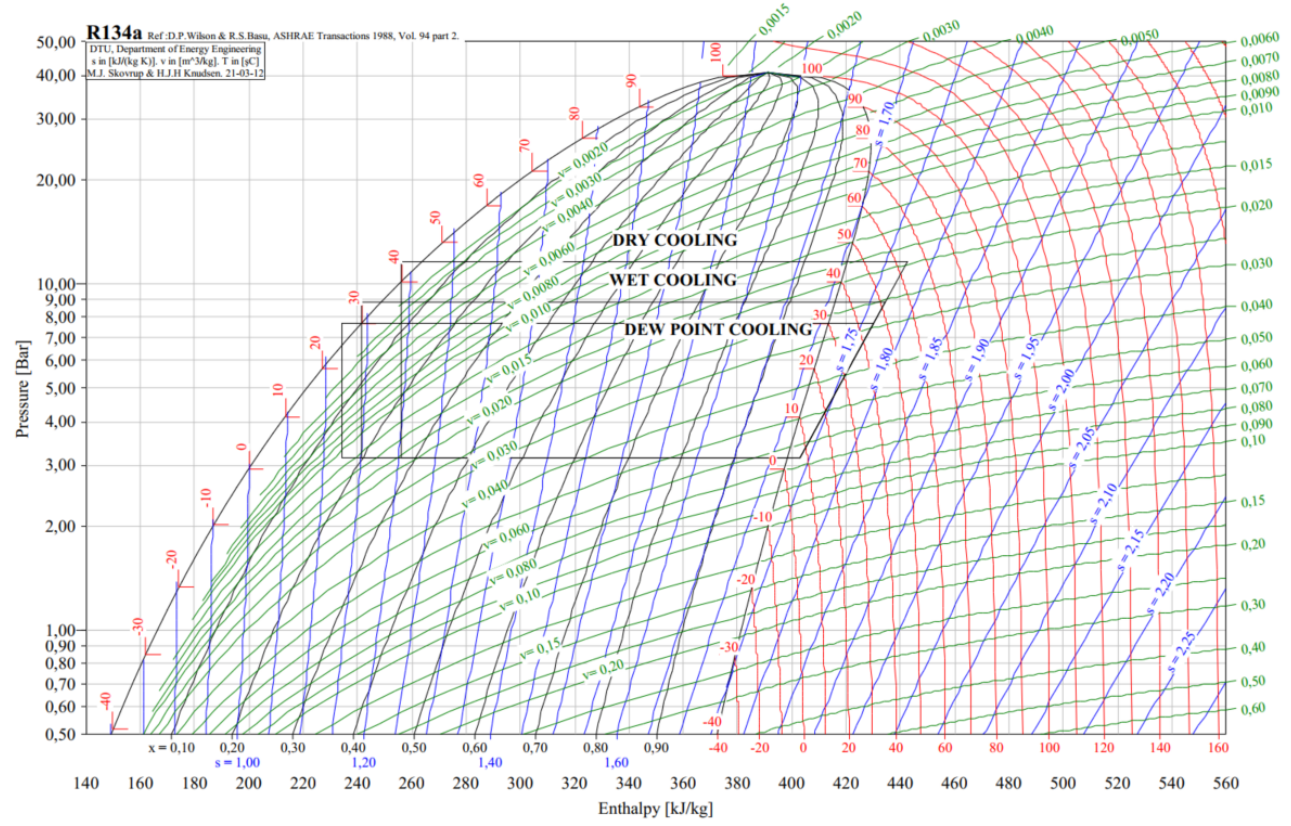

Fig. 13. Three different cooling solutions on the lgp-h diagram showing their cooling limits [9].

\section{Conclusions}

Nowadays most HVAC projects for comfort and industry are based on compressor cycle with air-cooled condensers. This solution is simple in terms of connections to each modules. Most commonly used are chillers which are delivered in a package, as plug and play solution. This solution is "on stock" which simplifies installation but not always puts enough effort into energy savings. Each installation should be investigated in much more ways than just simplification.

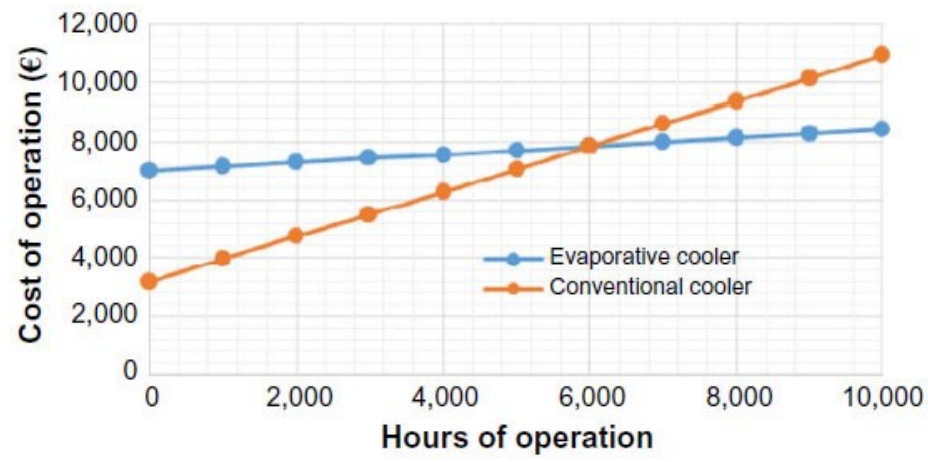

Fig. 14. The operational cost of an evaporative cooler and conventional cooler [12].

More effectiveness installation, for example with cooling towers, is less likely to use. It is mainly by they consume some water which additionally has to be treated and parameters kept in constant parameters. Even investment, time and cost, will bring quick savings. This was calculated by [12] and it is shown in Figure 14.

Connection of HMX as air precooling which enters cooling tower should be investigated and test in real ambient conditions. Reaching dew point at highest ambient conditions gives 
additional saving. It could be concluded, by the above shown preliminary investigations, that lowering reference temperature from wet-bulb up to dew point has a positive impact on energy consumption (directly and indirectly) and also water usage.

Fact that climate is changing should put more effort into finding and combining the most effective solutions like described in this paper IEC with DEC. This paper gives foundations for future scientific research. Connection of M-Cycle with the cooling tower will be made. Results will be for the polish climate which gives an idea of how much savings (on energy and water) we could obtain.

\section{Nomenclature}

$T_{E}$-evaporation temperature,

$Q_{E}-$ cooling capacity,

$T_{C}$-condensation temperature,

$Q_{C}$-heating capacity,

$W$ - compressor input,

$m$ - mass flow of refrigerant,

$\mathrm{C}$ - concentration cycle.

$V_{w}$ - evaporation loss

$Q_{r e f}$ - cooling capacity of the cooling tower

Subscrips:

wb - wet bulb

$\mathrm{db}-$ dry bulb

$\mathrm{c}-$ condenser/condensation

$\mathrm{e}$ - evaporator/evaporation 


\section{References}

1. Energy Market Agency S.A, Statistics Poland, Enterprises Department, Consumption of fuels and energy carriers in 2019, Warsaw (2020)

2. European Union, Reference Document on the application of Best Available Techniques to Industrial Cooling Systems, Ministerstwo Środowiska, Warszawa, January (2004)

3. MITA Cooling Technologies S.r.1.,Via del Benessere, 13, Siziano 27010, Pavia, IT, https://www.mitacoolingtechnologies.com/

4. D. Pandelidis, S. Anisimov, Energy Convers Manag 2015;90:62-83.

5. S. Anisimov, D. Pandelidis, J. Danielewicz., Energy 2015;80:452-464

6. S. Anisimov, D. Pandelidis, A. Jedlikowski, V. Polushkin, Energy 2014;76:593-606.

7. F. Scuderi, M. Proietti, N. De Cardenas, A. Fontana, P. Montrasio, A. Visintini, Saving resources by going evaporative: how evaporative cooling technologies and water cooled chillers can significantly reduce energy demands, AiCARR, Matera, May (2017)

8. Eurovent 80 Bd. A. Reyers Ln., 1030 Brussels, Belgium, Eurovent Rec 9/13 - 2019, December (2019)

9. CoolPack Version 1,50, IPU \& Department of Mechanical Engineering, Technical University of Denmark, 2000-2012, https:/www.ipu.dk/

10. Munters Sp. z o.o., Oddział w Polsce, ul. Świętojańska 45/17, 81-391 Gdynia, www.munters.com

11. M. H. Mahmood, M. Sultan, T. Miyazaki, S. Koyama, V. Maisotsenko, Renewable and Sustainable Energy Reviews 66 (2016) 537-555, 25 August (2016)

12. E. D. Rogdakis, D. N. Tertipis, Energy and Emission Control Technologies, Volume 2015:3 Pages 15-22, 6 March (2015) 